\title{
Not all that's new and shiny is good for healthcare
}

\author{
Fiona Godlee editor, BMJ
}

Concern has been growing about the use of metal on metal hip implants. In its 2011 report, the National Joint Registry for England and Wales identified problems including higher revision rates compared with more traditional ceramic or polyethylene implants (BMJ 2011;343:d5977). A BMJ investigation by Deborah Cohen asked why it took so long for one metal on metal implant, the ASR, to be withdrawn, highlighting denials and delays by the manufacturer and failings in the regulation of medical devices (BMJ 2011;342:d2905).

This week we publish a systematic review of research into the safety and effectiveness of hip implants. Art Sedrakyan and colleagues conclude that the evidence is inadequate, but what there is shows no advantage of metal on metal implants (or indeed ceramic on ceramic) compared with traditional metal on polyethylene implants (doi:10.1136/bmj.d7434). They also confirm the registry's findings of substantially higher rates of revision surgery with metal on metal implants.

What then should you do for patients with a metal on metal hip implant who develop symptoms suggesting joint failure? As Camden Fary and colleagues say (doi:10.1136/bmj.d7441), this is likely to be an increasingly common scenario as patients with silent or symptomatic adverse reactions to the metal wear debris start to seek help. They will often be younger and more active than most hip transplant patients and should be referred without delay for specialist investigation and imaging. Revision surgery is the most likely outcome. Unfortunately, in these circumstances, revision has worse outcomes than for other indications owing to inflammatory destruction of soft tissue and bone, and complication rates are higher. The authors conclude that this makes early identification and referral all the more crucial.
This saga sends a warning bell about the risks of innovation in healthcare. Not all that is new and shiny is good for health. As a further illustration, Wendy Lipworth and colleagues present the curious story of chronic tophaceous gout (doi:10.1136/bmj. d7459). This is the commonest inflammatory arthritis in older men, affecting $1-2 \%$ of adults in the developed world, and we have good treatments for it-allopurinol or probenecid, plus colchicine to prevent initial flare ups. Yet these safe, effective, and cheap drugs are underused. In the case of allopurinol, it is often prescribed at too low a dose, perhaps because of exaggerated fears of allopurinol hypersensitivity syndrome, causing apparent treatment failure. Meanwhile a far more expensive alternative, feboxostat, is overused, they say, and two years ago a company was granted a patent for colchicine, which has been available off patent for over a century. Curious indeed.

Health systems too can "innovate" in ways that impair rather than improve healthcare. Mark Welfare finds no merit in NICE's recommendation that all patients admitted to hospital should be assessed for risk of thromboembolism (doi:10.1136/bmj.d6452). "It may save no patients' lives but will cost millions of pounds," he says.

We hope for better contributions to improving health from the nominees and eventual winners of this year's BMJ Group Awards (doi:10.1136/bmj.d7958). New categories include Clinical Commissioning Team of the Year and the Karen Woo Award for the doctor who has gone well beyond the call of duty to care for patients. We greatly look forward to your nominations.

Cite this as: BMJ 2011;343:d7997

๑ BMJ Publishing Group Ltd 2011 